\title{
THREE-PARAMETER FEEDBACK CONTROL OF AMORPHOUS RIBBON MAGNETIZATION
}

\author{
Oleksandr Stupakov ${ }^{*}$ - Peter Švec ${ }^{* *}$
}

\begin{abstract}
This work describes a specially developed software for controllable magnetic hysteresis measurements of amorphous and nanocrystalline ribbons. The sophisticated algorithm enables to simplify a hardware design and to suppress an influence of experimental conditions on the measurement results. The main software feature is a three-stage feedback algorithm, which accurately adjusts the magnetization conditions: magnetization amplitude, geomagnetic bias and magnetization waveform. Air flux compensation of the induction signal is also performed by the software using an effective value of the coil cross section obtained from a calibration measurement without the ribbon. Applicability of the designed setup is illustrated for a series of nanocrystalline Hitperm ribbons measured at the power-line conditions: $50 \mathrm{~Hz}$ frequency and sinusoidal magnetization waveform.

Keywords: magnetic variables measurement, magnetic hysteresis, digital feedback control, amorphous magnetic materials
\end{abstract}

\section{INTRODUCTION}

Iron-based amorphous ribbons prepared by a rapid melt-spinning solidification demonstrate unique magnetic properties. Their disordered atomic arrangement does not prevent the formation of ferromagnetic order with high permeability and coercivity as low as a few A/m. Electrical resistivity of these glass metals is also $2-3$ times higher than that of their crystalline counterparts. These excellent characteristics make the amorphous magnetic alloys to be a potential candidate for replacing traditional electrical steels in a range of power devices, eg distribution transformers. However, at present the amorphous materials comprise only a few percents of world production of soft magnetic materials. Complicated preparation technique, high stress sensitivity of the magnetic properties and low saturation magnetization impede the implementation of the amorphous materials into a practice [1-3]. Therefore, a special attention is currently riveted on recently developed alloys with high saturation induction of up to $1.6-1.8 \mathrm{~T}[4]$.

Industrial application of these materials requires a suitable technique for quality testing. However, the accurate magnetic measurement of the soft amorphous ribbons with a tiny cross section and the near-zero coercivity is not an easy task. There is still no internationally agreed standard method, simple and reliable in practical use [2]. Tape-wound ring samples are preferable for the physically accurate measurement in a magnetically closed configuration. However, the tape winding is time consuming and implies the buildup of stresses, which can considerably vary the magnetic properties of the amorphous materials $[1,3,4]$. Therefore, the more practical solution is to magnetize the ribbons in a solenoid or Helmholtz coils.
This enables to calculate the magnetic field accurately using the magnetizing current because the demagnetizing factor of long and slim ribbons is negligible [5]. However, the measurements in these open magnetic configurations have some serious drawbacks: weak induction and considerable air flux signals, significant geomagnetic bias and uncontrollable magnetization waveform $[6,7]$. Therefore, to simplify and stabilize the measurement conditions, the experiments are often performed at a dc regime [5-7] or with oversaturated field amplitudes [3].

Modern digital methods of measurement and data processing afford new opportunities for development of the magnetic testing systems. Many hardware/analogue components can be easily substituted by the digital processing units providing more controllable experimental conditions and higher result accuracy [8-10]. This work describes such a new setup with a specially designed controlling software for the accurate magnetic hysteresis measurement of the amorphous ribbons at the standard conditions of transformer steel operation: $50 \mathrm{~Hz}$ sinusoidal magnetization with fixed amplitude $[1,11]$. The developed measurement software iteratively adjusts the magnetization conditions in three interconnected steps to symmetrize the hysteresis loop and to keep the required shape of the magnetization waveform. The setup is realized by analogy with our previous system developed for the measurements of bulky sheets of electrical steels in pseudoclosed magnetic configurations with a simpler two-stage control of the magnetization conditions [11]. The particular measuring and technical problems, which are important for further development of the field, are discussed in detail. The setup efficiency is proved by the experimental data.

\footnotetext{
* Institute of Physics, Academy of Sciences of the Czech Republic, v.v.i., Na Slovance 2, 18221 Prague, Czech Republic, stupak@fzu.cz; ** Institute of Physics, Slovak Academy of Sciences, Dúbravská cesta 9, 84511 Bratislava, Slovakia, peter.svec@savba.sk
} 


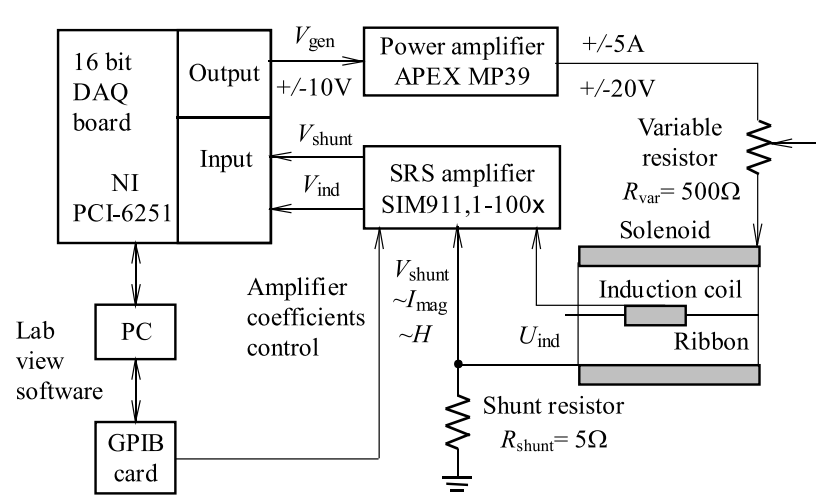

Fig. 1. Block scheme of the measurement setup

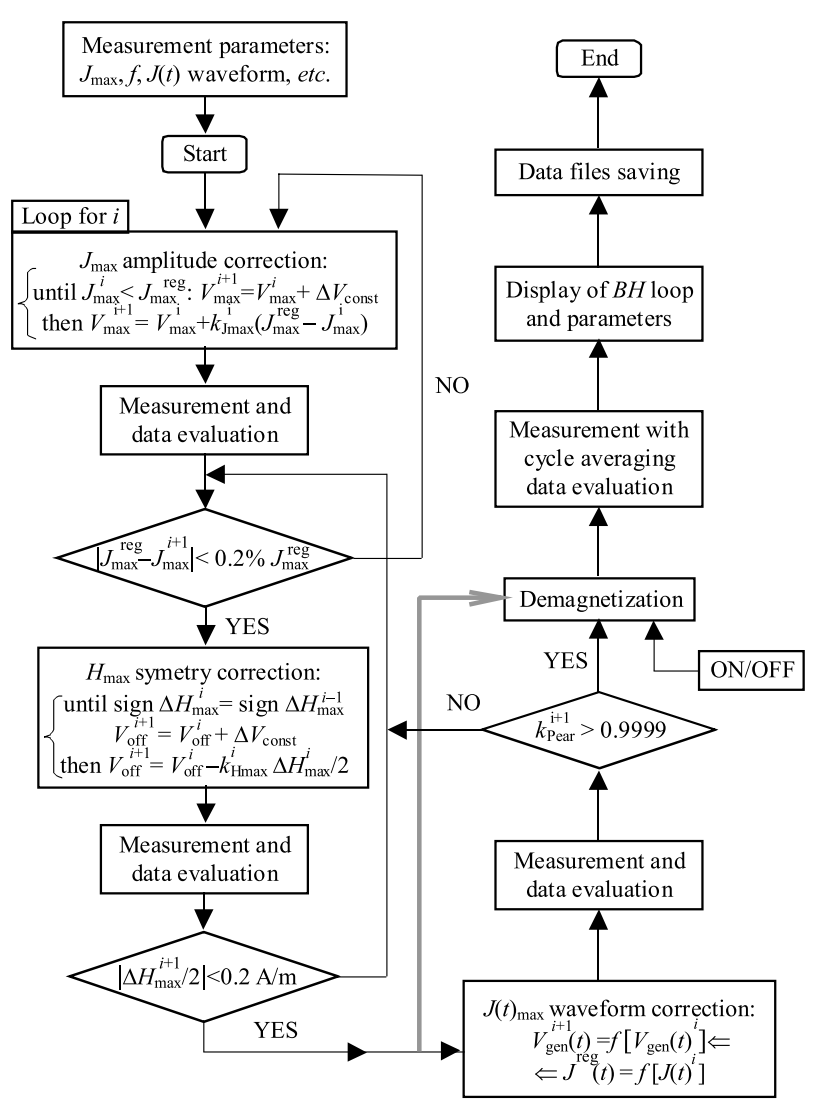

Fig. 2. Flowchart of the measurement algorithm

\section{SETUP DESCRIPTION}

The block scheme of the measurement setup is shown in Fig. 1. The measurements are PC-controlled through the 16-bit data acquisition board and a GPIB card. The magnetization voltage $V_{g e n}$ is generated by the acquisition board and processed by a power amplifier [11]. The ribbons are magnetized in a solenoid with a $4 \Omega$ active resistance. A power film resistor $R_{\text {shunt }}=5 \Omega$ mounted on a heat sink is used for the accurate reading of the voltage $V_{\text {shunt }}$ needed for determination of the ribbon magnetic field

$$
H=\frac{N}{l} I_{\text {mag }}=\frac{N}{l} \frac{V_{\text {shunt }}}{R_{\text {shunt }}}
$$

where $N$ is the number of turns of the solenoid magnetizing winding, $l=300 \mathrm{~mm}$ is the solenoid length and $I_{m a g}$ is the magnetization current. The specific number of the solenoid $N / l=4650 \mathrm{~A} / \mathrm{m}$ was also verified by a commercial Hall-effect gaussmeter. This widely accepted current field approach supposes that the inside-ribbon magnetic field is equal to the homogeneous field of empty solenoid. The demagnetizing factor of $\sim 1 \mathrm{~m}$ long and slim ribbons is negligible [5]. An additional variable resistor $R_{\text {var }} \simeq 500 \Omega$ is needed for full use of the output voltage range.

One induction coil of $40 \mathrm{~mm}$ length is used for determination of the ribbon magnetization. A calibration measurement of air flux signal without the sample replaces a common analogue method of two coil compensation. The sample was placed into one of these coils connected in series opposite. However, an additional balance unit was usually needed for perfect compensation of the air induced signal [6,7]. According to the Faraday's law [1], the voltage $U_{\text {ind }}$ induced in the coil encircling the ribbon is equal to

$$
U_{i n d}=-n S \frac{\mathrm{d} B}{\mathrm{~d} t}=-n\left[S_{c o i l} \mu_{0} \frac{\mathrm{d} H}{\mathrm{~d} t}+S_{r i b} \frac{\mathrm{d} J}{\mathrm{~d} t}\right],
$$

where $n=2700$ is the number of turns of the induction winding, $B$ is the magnetic induction, $t$ is time, $\mu_{0}=$ $4 \pi 10^{-7} \mathrm{~N} \mathrm{~A}^{-2}$ is the permeability of vacuum, $J$ is the magnetization, $S_{\text {coil }}$ and $S_{r i b}$ are the cross sections of the induction coil and the ribbon, respectively. Numerical trapezoidal integration of the sampled induction voltage gives

$$
\int U_{i n d} \mathrm{~d} t=-n\left[S_{\text {coil }} \mu_{0} H(t)+S_{\text {rib }} J(t)\right]
$$

The first air induced component $n S_{\text {coil }} \mu_{0} H(t)$ of (3) is pre-measured without the sample to determined the effective coil cross section $S_{\text {coil }}=88 \mathrm{~mm}^{2}$ (inner and outer coil cross-sections are $3 \times 10$ and $9 \times 17 \mathrm{~mm}^{2}$ ). Then the ribbon magnetization is easily calculated using the formula

$$
J(t)=\frac{-1}{n S_{\text {rib }}}\left[\int U_{\text {ind }} \mathrm{d} t+n S_{\text {coil }} \mu_{0} \frac{N}{l} \frac{V_{\text {shunt }}(t)}{R_{\text {shunt }}}\right] .
$$

Because of the accommodation effect, the signals are collected and integrated from the fifth measurement cycle. The constant zero threshold of $U_{\text {ind }}$ is compensated for by levelling the magnetization amplitudes $J_{\max }$, and the $J(t)$ waveform is symmetrized around zero.

The software was realized in a NI LabVIEW graphical programming environment; the algorithm flowcharts are shown in Figs. 2 and 3. The main algorithm feature is the three-stage feedback control of the magnetization conditions. At the first stage, the magnetization amplitude $J_{\max }$ is iteratively adjusted with $0.2 \%$ accuracy by selecting an appropriate amplitude $V_{\max }$ of the generated sinusoidal voltage $V_{g e n}$. After the $J_{\max }$ adjustment, 


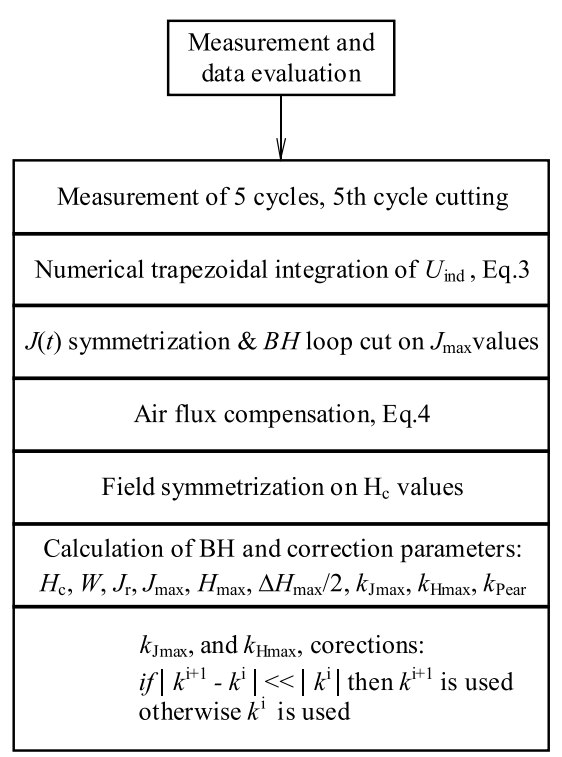

Fig. 3. Detailed scheme of the measurement subroutine shown in Fig. 2

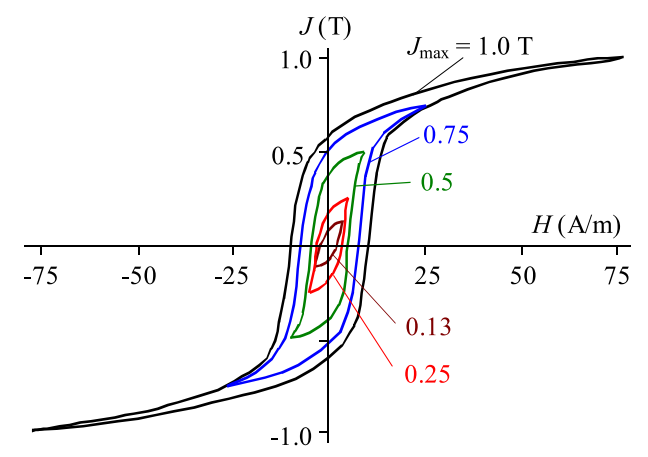

Fig. 4. Set of the symmetrized hysteresis loops with different magnetization amplitudes $J_{\max }$. The measurements were performed for the softest $\mathrm{Fe}_{60.7} \mathrm{Co}_{20.3} \mathrm{Nb}_{7} \mathrm{~B}_{12}$ ribbon at the conditions of $50 \mathrm{~Hz}$ magnetization without the $J(t)$ waveform control.

the correction coefficient $k_{J \max }=d V_{\max } / d J_{\max }$ describing the rate of change of the generated voltage amplitude with respect to the magnetization amplitude is stored for further $J_{\max }$ corrections.

After the symmetrization of the obtained hysteresis loops $J(H)$ on the coercive force values $H_{c}$, there is a substantial asymmetry with respect to the field amplitudes $\Delta H_{\max } / 2 \simeq 10-20 \mathrm{~A} / \mathrm{m}$. This field shift, which is mostly caused by the Earth's magnetic field, leads to a significant distortion of the narrow hysteresis loops with $H_{c} \simeq 5-10 \mathrm{~A} / \mathrm{m}$. This field distortion is much more significant than for the previously considered case of electrical steels measured in quasi-closed magnetic configurations [11]. Therefore, its correction should be performed on a more serious base, ie it should be realized inside a main feedback loop. So at the second stage, the field amplitudes $H_{\max }$ are similarly symmetrized with $\pm 0.2 \mathrm{~A} / \mathrm{m}$ accuracy by selecting an appropriate offset of the generated voltage waveform, $V_{\text {gen }}(t)=V_{\max } \sin (\omega t)+V_{\text {off }}$. After the $H_{\max }$ symmetrization, the second correction coefficient $k_{H \max }=\mathrm{d} V_{o f f} / \mathrm{d}\left(\Delta H_{\max } / 2\right)$ describing the rate of change of the voltage offset with respect to the field amplitude shift is also stored.

The controls of $J_{\max }$ and $\Delta H_{\max } / 2$ parameters are interrelated: the change of magnetization amplitude alters the field symmetry and vice versa. Therefore, for the simultaneous multi-parameter control, the feedback loop iterations should be combined with the consecutive control of each magnetic parameter. After the initial corrections of $J_{\max }$ amplitude and $H_{\max }$ symmetry, such an iterative feedback loop is initiated. The $J_{\max }$ amplitude is checked first: if its value differs from the required one by more than $0.2 \%$, the $k_{J \max }$ coefficient is used to correct the $V_{\max }$ level. If $J_{\max }$ is within the tolerance limit, then the $\left|\Delta H_{\max } / 2\right|$ parameter is checked to be less than $0.2 \mathrm{~A} / \mathrm{m}$ and can be corrected using the $k_{H \max }$ coefficient. After each parameter correction, the hysteresis loop is remeasured and new $J_{\max }$ and $\Delta H_{\max } / 2$ parameters together with the correction coefficients $k_{J \max }$ and $k_{H \max }$ are recalculated at the end of each feedback cycle (see Figs. 2 and 3 for more details). After full simultaneous adjustment of $J_{\max }$ and $\Delta H_{\max } / 2$ parameters, the final hysteresis measurement is performed in the "common" way without the $J(t)$ waveform control for comparison with the measurement at the standard operation conditions with the controlled $J(t)$ (gray arrow labeled as "Measurement without $J(t)$ control" in Fig. 2). At these measurement conditions (without the $J(t)$ control and with high $R_{v a r}$ used), the resistive part of the magnetization circuit resistance is dominant over the inductive part and the open magnetic configuration of solenoid keeps the field waveform to be practically sinusoidal, $H(t) \sim I_{\text {mag }} \sim V_{\text {gen }} \sim \sin (\omega t)$ [3]. Example of such measurements is shown in Fig. 4 for visual illustration of the efficiency of the used symmetrization algorithm.

For the measurements with the required magnetization waveform $J(t)$, the third control subroutine is added to the main feedback algorithm (see Fig. 2). If the first two parameters $J_{\max }$ and $\Delta H_{\max } / 2$ have been adjusted within the tolerance limits, the measured magnetization waveform $J(t)$ is compared with the required (sine) waveform $J(t)^{r e q}$. For numerical evaluation of the waveform coincidence, a Pearson's correlation coefficient [11] is used

$$
k_{\text {Pear }}=\frac{\sum_{j}\left(J_{j}-\langle J\rangle\right)\left(J_{j}^{r e q}-\left\langle J^{r e q}\right\rangle\right)}{\sqrt{\sum_{j}\left(J_{j}-\langle J\rangle\right)^{2} \sum_{j}\left(J_{j}^{r e q}-\left\langle J^{r e q}\right\rangle\right)^{2}}}
$$

where the summation is given over the sampled points $j$. The acceptable level of waveform convergence is usually set in the range of $k_{\text {Pear }}=0.9999-0.99995$, which corresponds to the form factor of $1.11 \pm 0.5 \%$. If the Pearson's coefficient $k_{\text {Pear }}$ is lower than the prescribed limit, a simple routine of the signal amplitude re-sampling is launched. The routine principle is intuitive: the adjusted waveform of the generated voltage $V_{g e n}\left(t_{j}\right)^{i+1}$ at the next $(i+1)$-th iteration step is calculated by time re-sampling 


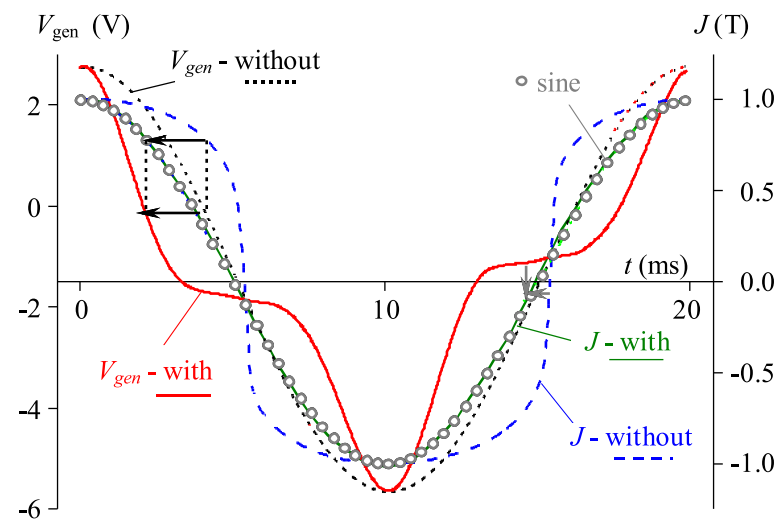

(a)

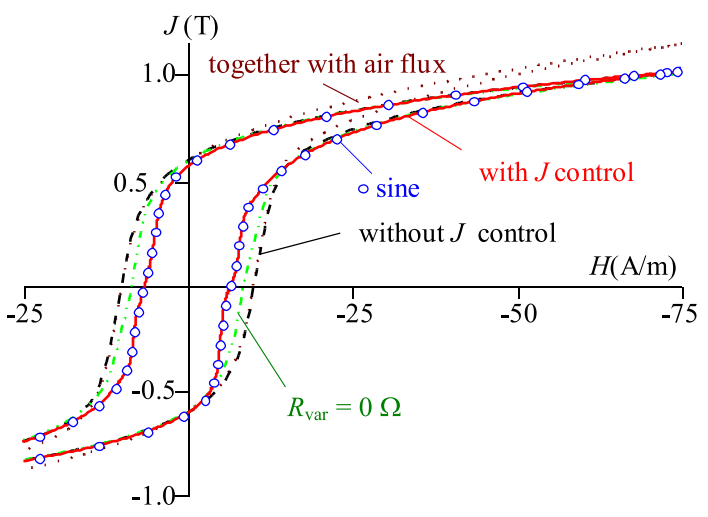

(b)

Fig. 5. (a) - Waveforms of the generated voltage $V_{\text {gen }}$ (left axis) and the corresponding magnetization $J$ (right axis) for the measurements performed with and without the $J$ waveform control. Principle of the $V_{g e n}$ re-sampling is illustrated by black arrows, (b) - The corresponding hysteresis loops measured with and without the $J$ waveform control. The loops obtained without the air flux compensation and without the variable resistor $R_{v a r}=0 \Omega$ are shown for the case of uncontrolled $J$ waveform. The required sinusoidal waveforms are shown by round symbols for comparison. The arrows present the region of steep signal changes, which is problematic for the $J$ waveform adjustment. The measurements were performed for the same softest ribbon at the conditions of $50 \mathrm{~Hz}$ magnetization and $J_{\max }=1 \mathrm{~T}$.

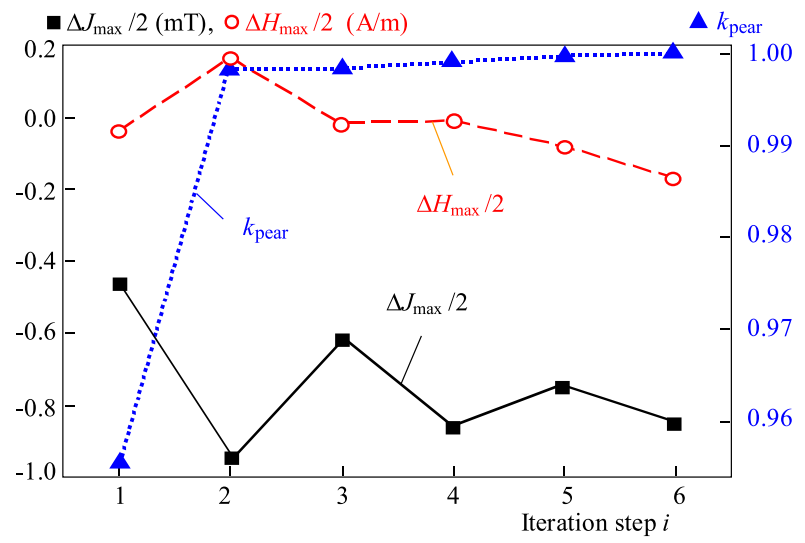

Fig. 6. Convergence of the three-parameter digital feedback algorithm: variations of the magnetization amplitude deviation from the required value $\Delta J_{\max }=J_{\max }-J_{\max }^{r e q}$ (divided by 2 for better visual presentation), of the field amplitude asymmetry $\Delta H_{\max } / 2$ (left axis) and of the Pearson's correlation coefficient $k_{\text {Pear }}$ (right axis) with the feedback iterations. The measurement was performed for the same ribbon and the same magnetization conditions.

of the voltage function $V_{g e n}\left(t_{j}\right)^{i}$ used at the previous $i$-th iteration step the way the corresponding measured magnetization waveform $J\left(t_{j}\right)^{i}$ is similarly time re-sampled to a required (sine) function $J\left(t_{j}\right)^{r e q}[11,12]$.

The graphical illustration of this routine is shown in Fig. 5(a) by black arrows. The figure also demonstrates the significant offset of the generated voltage waveform $V_{\text {off }} \simeq-1.4 \mathrm{~V}$ applied for the $H_{\max }$ symmetrization. However, it should be noted that such a high $V_{\text {off }}$ value corresponds to the maximal value of the Earth's magnetic field of $\sim 25 \mathrm{~A} / \mathrm{m}$ measured by a conventional gaussmeter along the solenoid axis. These values are in full agreement with (1): $V_{\text {off }} \simeq H_{\text {Earth }} R_{\text {var }} / 2(N / l)=1.34 \mathrm{~V}$ (2 in denominator is a coefficient of the power amplifier). The dc magnetic bias can be easily decreased up to $\sim 5-10 \mathrm{~A} / \mathrm{m}\left(V_{\text {off }} \simeq 0.25-0.5 \mathrm{~V}\right)$ by a suitable positioning of the solenoid along the geomagnetic field lines and a partial shielding of the solenoid side faces. By putting the solenoid inside a transformer steel box, the level of dc bias can be further decreased to an almost negligible value of $\sim 1 \mathrm{~A} / \mathrm{m}$; however, this construction is not suitable for on-line industrial testing or quick sample replacement. Figure 5(b) presents the corresponding hysteresis loops measured with and without the $J(t)$ waveform control. It is well seen that the sinusoidal control of the $J(t)$ waveform gives the considerably narrower loop. However, the loop shape does not change in the saturation region, where the magnetic permeability is low. The measurement without the $J(t)$ control and with minimum active resistance of the magnetization circuit (the variable resistance $R_{v a r}$ is set to zero) makes the magnetization conditions closer to the sinusoidal $J(t)$. However, it is well seen that the open magnetic configuration of the solenoid cannot automatically keep the sinusoidal induction signal $U_{\text {ind }} \sim \sin$ at power line magnetization frequency [11]. The hysteresis loop measured without the air flux compensation is also shown for comparison.

The described feedback algorithm usually converges after several tens of cycles. The initial blind adjustments of $J_{\max }$ and $\left|\Delta H_{\max } / 2\right|$ parameters require approximately ten iterations each. Then the three-parameter feedback routine also needs approximately ten cycles for final accurate adjustment of the magnetization conditions. This is illustrated by Fig. 6: $J_{\max }$ deviation is within the tolerance limit of $\pm 2 \mathrm{mT}\left(0.2 \%\right.$ of $J_{\max }=$ $1 \mathrm{~T})$; the first iteration significantly changes the $J(t)$ waveform - $k_{\text {Pear }}$ rapidly increases from 0.95524 to 0.99812 ; the second iteration corrects the $H_{\max }$ symmetry; the subsequent three iterations gradually adjust the $J(t)$ waveform to the final $k_{\text {Pear }}=0.99994$.

However, there are some important factors, which are critical for the algorithm convergence. First of all, the magnetization amplitude should be controlled precisely: two noticeable stairs in the $V_{\text {gen }}(t)$ waveform are generated at the next re-sampling iteration if the actual magnetization amplitude $J_{\max }$ is lower than the required 

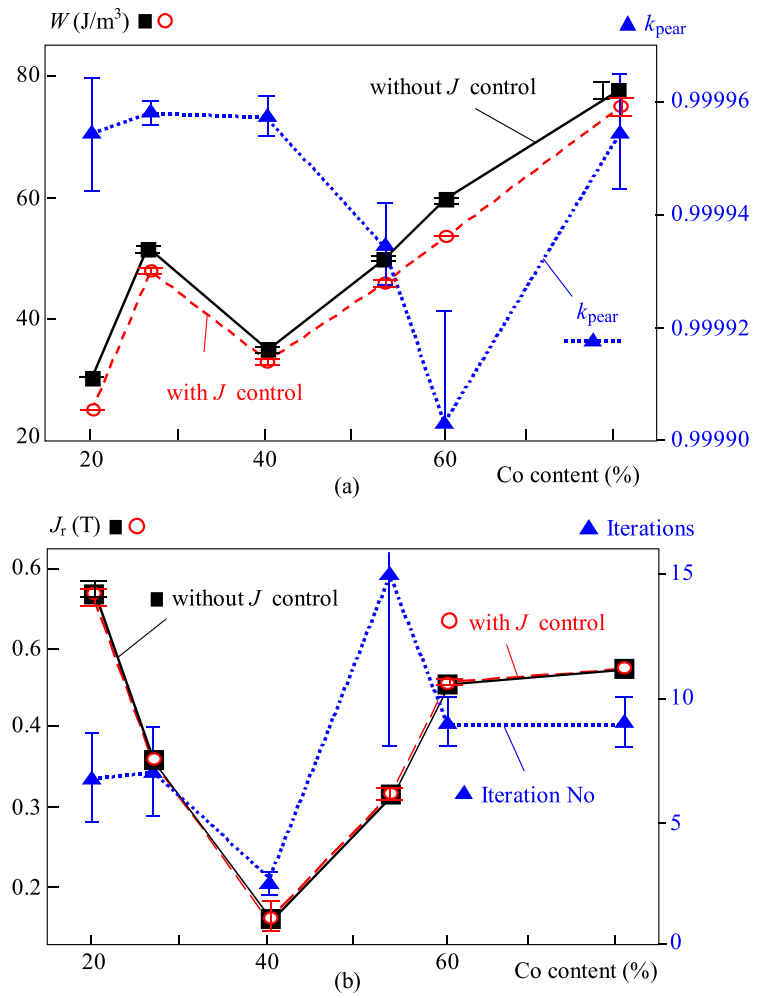

Fig. 7. Dependence of the classical hysteresis parameters: (a) - the hysteresis loss $W$, and (b) - the remanent magnetization $J_{r}$, on the Co content for the tested Hitperm series (left scales). The data obtained with and without the $J$ waveform control $\left(J_{\max }=1 \mathrm{~T}\right)$ are shown for comparison. The corresponding convergence characteristics of the developed feedback algorithm are presented on the right figure scales: the achievable Pearson's correlation coefficient: (a) $-k_{\text {Pear }}$, and (b) - the required number of iterations. The error bars are evaluated as the standard error of three identical tests at the different positions along the ribbons length.

one [11]. The next convergence problem is related to the algorithm complexity and high measurement accuracy. The magnetization conditions are very sensitive to the changes of experimental parameters. Therefore, it is important to control the obtained correction coefficients $k_{J \max }$ and $k_{H \max }$ at each iteration step. Additional conditional subroutines are developed to correct the coefficient values in order to avoid uncontrollable sudden changes of the magnetization conditions (see Fig. 3). However, these two problems can be settled. The principal issue is a general applicability of the used algorithm of the $J(t)$ adjustment, which is based on a simple correction of the $V_{\text {gen }}(t)$ signal amplitude only [13]. The main problem is to convert the vertical pulse of the initial $J(t)$ waveform, which is typical for the measurement in the magnetically open solenoid, to the required sinusoidal signal because this conversion results in a nearly constant $V_{\text {gen }}(t)$ signal (gray arrows in Fig. 5). For very soft amorphous ribbons with practically vertical $J(t)$ pulse, the horizontal step of the $V_{\text {gen }}(t)$ waveform cannot be generated with the required accuracy. In these local regions of the highest magnetization rate, it could be impossible to adjust the $J(t)$ waveform to the sinusoidal shape exactly. To increase the accuracy of the $J(t)$ waveform adjustment, the variable resistor $R_{\text {var }}=500 \Omega$ is used. This significantly in-
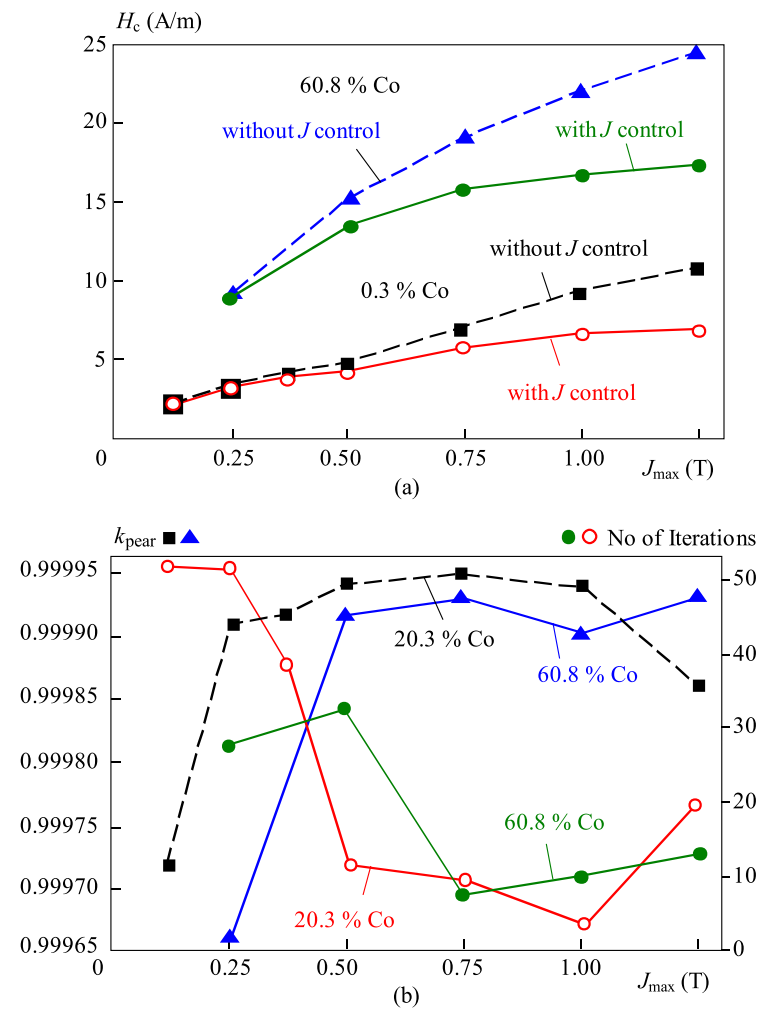

Fig. 8. (a) - Dependence of the classical coercive field $H_{c}$ on the magnetization amplitude $J_{\max }$ for the two samples with different Co content, $x=20.3$ and 60.8 at\%. The data obtained with and without the $J$ waveform control are shown for comparison, (b) the corresponding convergence characteristics of the digital feedback: the achievable Pearson's correlation coefficients $k_{P e a r}$ (left scale) and the required number of iterations (right scale). The measurements were performed at $50 \mathrm{~Hz}$ magnetization frequency.

creases the resolution of the adjusted output signal $V_{\text {gen }}$; in addition, the amplitude adjustment algorithm seems to converge better with higher active resistance of the magnetization circuit. The problem could be also solved by using more sophisticated and time-consuming feedback algorithms, eg an algorithm with the simultaneous corrections of $V_{g e n}$ amplitude and phase $[8,14]$.

Before the final measurement with the fitted $V_{\text {gen }}(t)$ signal, the demagnetization to the obtained geomagnetic offset $V_{\text {off }}$ can be performed. The magnetization $V_{\text {shunt }}$ and the induction $U_{\text {ind }}$ voltages are amplified with the GPIB adjusted coefficients, acquired through a board multiplexer at a sampling rate of $100-200 \mathrm{kHz}$ and onthe-fly averaged over adjacent data points to the final one thousand points for one magnetization cycle. Averaging over the consequent magnetization cycles can be performed for additional signal smoothing. At the condition of $50 \mathrm{~Hz}$ magnetization, the output voltage $V_{g e n}$ can be generated with the sampling rate of up to $100-$ $200 \mathrm{kHz}$, which is quite sufficient for a smooth signal. Data evaluation and saving are also performed by the software; all classical hysteresis parameters (coercive force $H_{c}$, hysteresis loss $W$, remanent magnetization $J_{r}$, field and magnetization amplitudes, $H_{\max }$ and $J_{\max }$ ) are calculated and stored. The measurement results are visu- 

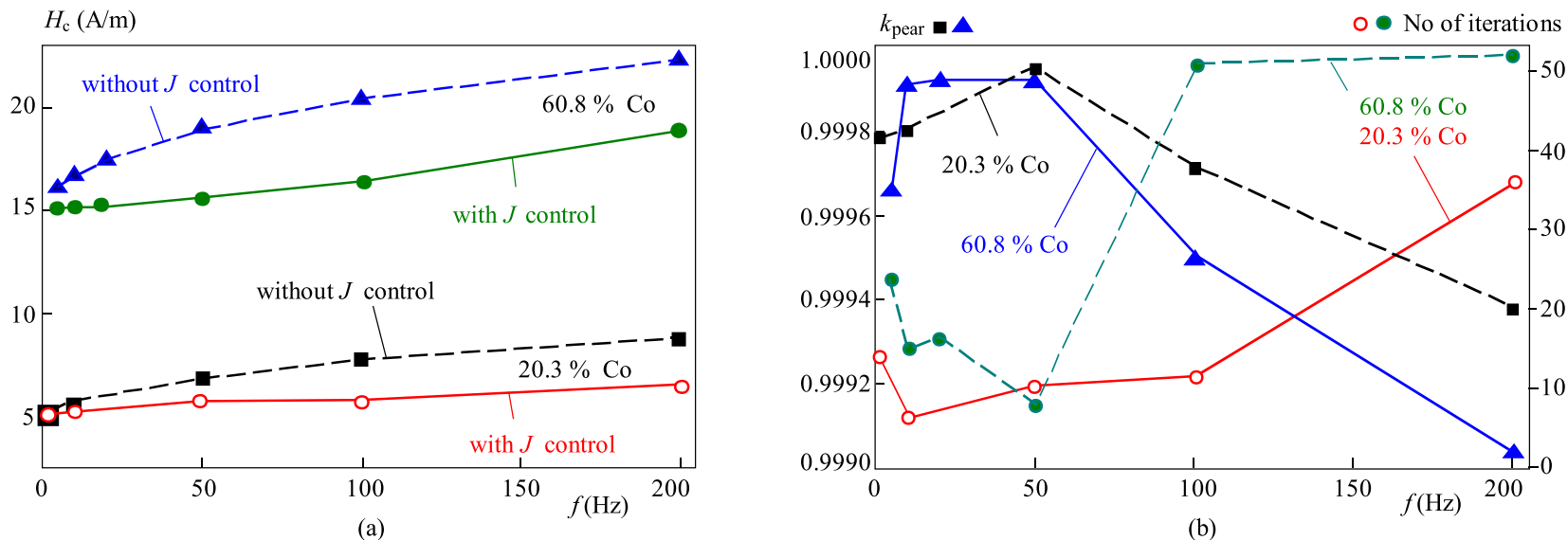

Fig. 9. (a) - Dependence of the classical coercive field $H_{c}$ on the magnetization frequency $f$ for the two samples with different Co content, $x=20.3$ and 60.8 at\%. The data obtained with and without the $J$ waveform control are shown for comparison, (b) - the corresponding convergence characteristics of the digital feedback: the achievable Pearson's correlation coefficients $k_{P e a r}$ (left scale) and the required number of iterations (right scale). The measurements were performed with the magnetization amplitude $J_{\max }=0.75 \mathrm{~T}$.

ally controlled in the LabVIEW graphic environment (see Figs. 2 and 3) [11].

\section{MEASUREMENT RESULTS}

In this section the efficiency of the developed setup is briefly illustrated and discussed on the basis of the characteristic experimental results. The results were shown for the series of as-cast nanocrystalline Hitperm ribbons $\mathrm{Fe}_{81-x} \mathrm{Co}_{x} \mathrm{Nb}_{7} \mathrm{~B}_{12} \quad(x=20.3,27,40.5,54,60.8$ and 81 at\%) [3]. The ribbons were at least $0.8 \mathrm{~m}$ long, $6-$ $8 \mathrm{~mm}$ wide and $\sim 10 \mu \mathrm{m}$ thick. All samples were tested at power line conditions: $50 \mathrm{~Hz}$ sinusoidal magnetization with two different amplitudes $J_{\max }=0.75$ and $1 \mathrm{~T}$. Each ribbon was measured at three different positions along its length: in the middle and $\pm 0.2 \mathrm{~m}$ from it. Two characteristic samples with $x=20.3$ and 60.8 at\% were also measured with different magnetization frequencies $f$ and amplitudes $J_{\max }$.

Dependence of the classical hysteresis loss $W$ and remanent magnetization $J_{r}$ for the tested ribbon series are shown together with the feedback convergence characteristics in Fig. 7. For the sake of simplicity, only the data measured with $J_{\max }=1 \mathrm{~T}$ are shown; the data obtained for the lower $0.75 \mathrm{~T}$ amplitude demonstrate similar relations with lower absolute values. Dependence of the coercive force is similar to that of the hysteresis loss; $H_{c}$ values are varied in the range of $6-22 \mathrm{~A} / \mathrm{m}$ (not shown here). The performed experiments have proved that the developed measurement system can stabilize the magnetization conditions of different amorphous ribbons with a high accuracy. From five to ten iterations are usually needed to adjust the $J(t)$ waveform to the required sinusoidal shape precisely with $k_{\text {Pear }}>0.9999$. The standard measurement error of three identical tests for the classical hysteresis parameters is also relatively low; it is in the range of $1-2 \%$ including the technological error of structure variations [15]. The $J(t)$ waveform control is important for accurate evaluation of the coercive force and the hysteresis loss, which are the main industrial parameters of the soft magnetic materials defining their efficiency to transfer the magnetic flux [11]. Figure 7(a) clearly demonstrates that the usual measurement methods without the accurate $J(t)$ control can give rather overestimated values of these characteristic parameters (see also Figs. 5(b), $8(\mathrm{a})$ and $9(\mathrm{a}))$.

Figures 8(a) and 9(a) present the dependence of the coercive force $H_{c}$, which is the most sensitive parameter with respect to the $J(t)$ waveform control, on the magnetization amplitude $J_{\max }$ and frequency $f$, respectively. Figures 8(b) and 9(b) show the corresponding convergence parameters of the developed feedback procedure: the achievable Pearson's correlation coefficient $k_{P e a r}$ and the required number of iterations. At low magnetization amplitudes and frequencies, the data obtained with and without the $J(t)$ control are very similar because of similar sinusoidal $J(t)$ waveforms and the near quasistatic magnetization, respectively. However, a weak induction signal makes difficulties for the feedback convergence: $k_{\text {Pear }}<0.9999$ and the iteration number tends to a predetermined limit of 50 cycles. For better convergence, the number of induction coil turns should be increased or an accurate analogue integrator (fluxmeter) should be used $[6,7]$. In addition, the measurements at low magnetization frequencies are more time-consuming. At higher magnetization amplitudes and frequencies, the difference between the data obtained with and without the $J(t)$ control becomes significant (see Figs. 8(a) and $9(\mathrm{a})$ ). The developed feedback system provides the best controllable conditions for the region of moderate magnetic saturation with well defined $H_{\max }$ peaks, $i e$ for the region of $J_{\max }=0.5-1 \mathrm{~T}$ in Fig. 8(b). At very high $J_{\max }$ values, the $J(t)$ waveform degenerates into a nearly constant signal, which leads to the above-mentioned convergence problem of the $J(t)$ adjustment procedure [13]. The $J(t)^{r e q}$ convergence quality becomes worse at high magnetization frequencies as well because of increasing inductive part of the magnetization circuit resistance, see 
Fig. 9(b). However, the setup is developed and optimized exactly for the measurements at power line magnetization frequency and industrially applicable magnetization amplitudes. In this range of standard experimental conditions, the setup does provide new performance capabilities.

\section{CONCLUSIONS}

The work described a novel setup for ac magnetic hysteresis measurements of the amorphous ribbons performed at controllable standard magnetization conditions: power line magnetization frequency and industrially applicable magnetization amplitudes. For this purpose, a three-stage controlling algorithm with digital feedback was developed. This algorithm sequentially adjusts three main parameters of the magnetization process: the magnetization and the field amplitudes together with the magnetization waveform. All the technical problems solved during the setup development as well as the software algorithm were analyzed in detail. Special attention was devoted to the question of general applicability of the algorithm used for the magnetization waveform control. It was shown that this simple method of signal amplitude adjustment can stabilize the measurements in the required range of the experimental conditions. However, more sophisticated and time-consuming algorithms should be generally developed and tested for accurate measurements in whole range of magnetization frequencies and at high saturation amplitudes. The efficiency of the proposed measurement method was illustrated on the series of nanocrystalline Hitperm ribbons tested at the power line frequency of $50 \mathrm{~Hz}$ and the sinusoidal magnetization waveform. The outcomes of this work are important for further unification of the measurement approaches and industrial online techniques.

\section{Acknowledgements}

The authors appreciates the financial support of the Czech Science Foundation (GACR) under the postdoctoral project No 102/09/P108 and that of the Slovak Science Agency (VEGA) under the grant No 2/0111/11.

\section{REFERENCES}

[1] FIORILLO, F.: Measurement and Characterization of Magnetic Materials, Elsevier Academic Press, Amsterdam, 2004.

[2] MOSES, A.: Iron-Based Amorphous Ribbons - Challenges and Opportunity for Power Applications, Journal of Optoelectronics and Advance Materials 4 (2002), 231-236.

[3] Butvin, P.-ButvinovÁ, B.-ŠVeC, P.-SiteK, J. : Application Potencial of Nanocrystalline Ribbons Still Pending, J. Electrical Engineering 61 (2010), 264-270.

[4] OHTA, M.-YOShIZAWA, Y.: Recent Progress in High $B_{s}$ Fe-Based Nanocrystalline Soft Magnetic Alloys, Journal of Physics D: Applied Physics, No. 44 (2011), 064004.
[5] PANDA, A.-BASU, A. MITRA, A. : Demagnetisation Effect and its Correction on the Measurement of Magnetic Hysteresis Loop of Melt-Spin Ribbons, Journal of Magnetism and Magnetic Materials 261 (2003), 190-195.

[6] SQUIRE, P.-SHEARD, S.-CARTER, C.-GIBBS, M. : Digital M-H Plotter for Low-Coercivity Metallic Glasses, Journal of Physics E: Scientific Instruments 21 (1988), 1167-1172.

[7] KULIK, T.-SAVAGE, H.-HERNANDO, A. : A High-Performance Hysteresis Loop Tracer, Journal of Applied Physics 73 (1993), 6855-6857.

[8] White, S.-KRAUS, T.-CLAPHAM, T.: A Multichannel Magnetic Flux Controller for Periodic Magnetizing Conditions, IEEE Transactions on Instrumentation and Measurement 61 (2012), 1896-1907.

[9] WAKABAYASHI, D.-TODAKA, T.-ENOKIZONO, M. : Measurement of Three-Dimensional Magnetostriction on GrainOriented Electrical Steel Sheet, J. Electrical Engineering 62 (2011), 153-157.

10] SOTO, M.-MARTINEZ-DE-GUERENU, A.-GURRUCHAGA, D.-ARIZTI, F. : A Completely Configurable Digital System for Simultaneous Measurements of Hysteresis Loops and Barkhausen Noise, IEEE Transactions on Instrumentation and Measurement 58 (2009), 1746-1755.

[11] STUPAKOV, O.: System for Controllable Magnetic Measurement with Direct Field Determination, Journal of Magnetism and Magnetic Materials 324 (2012), 631-636.

[12] BERTOTTI, G.-FERRARA, E.-FIORILLO, F.-PASQUALE, M.: Loss Measurements on Amorphous Alloys under Sinusoidal and Distorted Induction Waveform using a Digital Feedback Technique, Journal of Applied Physics, 73 (1993), 5375-5377.

[13] STUPAKOV, O.: Controllable Magnetic Hysteresis Measurement of Electrical Steels in a Single-Yoke Open Configuration, IEEE Transactions on Magnetics, doi: 48 (2012), 4718-4726.

14] ZUREK, S.-MARKETOS, P.-MEYDAN, T.-MOSES, J. : Use of Novel Adaptive Digital Feedback for Magnetic Measurements under Controlled Magnetizing Conditions, IEEE Transactions on Magnetics 41 (2005), 4242-4249.

15] PETRIDIS, C.-KTENA, A.-BOLSHAKOVA, I.-HRISTOFOROU, E. : On the Magnetic and Magnetoelastic Uniformity Measurements on Magnetostrictive Ribbons and Wires, Journal of Magnetism and Magnetic Materials 316 (2007), e628-e631.

Received 14 June 2012

Oleksandr Stupakov $(\mathrm{PhD})$ received his MSc degree in Physics from Donetsk State University, Ukraine, in 1999 and PhD degree in Physics of Condensed Matter and Material Research from Charles University in Prague, Czech Republic, in 2006. From 2006 to 2008, he was a JSPS postdoctoral fellow at Institute of Fluid Science, Tohoku University, Japan. At present he is a scientist at Institute of Physics, Czech Academy of Sciences. He is the co-/author of $>25$ papers. His fields of interest are non-destructive evaluation of structural materials by magnetic inductive methods; stabilization and unification of magnetic hysteresis and Barkhausen noise techniques; measurement systems and digital signal processing.

Peter Švec (Ing, DrSc), born in Bratislava, Czechoslovakia, in 1955, graduated from the Faculty of Electrical Engineering, Slovak University of Technology, Bratislava, from Solid State Physics branch, in 1979, and received the PhD degree in Physics in 1986. At present he is with the Institute of Physics, Slovak Academy of Sciences. His research field is the physic of metals, recently namely phase transformations in metal-metalloid glassy systems. 